Communications in Physics, Vol.22, No. 1 (2012), pp. 53-58

\title{
EFFECT OF THE DIRECT EXCHANGE INTERACTION BETWEEN MAGNETIC IMPURITIES ON MAGNETIZATION IN DILUTED MAGNETIC SEMICONDUCTORS
}

\author{
VU KIM THAI \\ Institute of Physics, VAST \\ LE DUC ANH \\ Hanoi National University of Education \\ HOANG ANH TUAN \\ Institute of Physics, VAST
}

\begin{abstract}
We consider a model of III-V diluted magnetic semiconductors where both of the exchange interaction between carrier and impurity spins, and the direct exchange interaction between magnetic impurities are taken into account. The magnetization as a function of temperature for a wide range of model parameters is calculated and discussed. We show that for a degenerate carrier system the suppression of the magnetization is sensitive to the antiferromagnetic coupling constant and the impurity concentration.
\end{abstract}

\section{INTRODUCTION}

The DMS combine ferromagnetism with the conductivity properties of semiconductors. Therefore, they are ideal materials for applications in spintronics where not only the electron charge but also the spin of the charge carrier is used for information processing. For instance, they allow to resolve the conductivity mismatch problem which hinders a high polarizability of injected electrons in a ferromagnetic metal/semiconductor junction [1].

One prominent DMS is $\mathrm{Ga}_{1-x} \mathrm{Mn}_{x}$ As (typical $x \approx 1-10 \%$ ) with the $\mathrm{Mn}$ ions substitutionally replacing $\mathrm{Ga}$ at the cation sites. Mn ions in $\mathrm{Ga}_{1-x} \mathrm{Mn}_{x} \mathrm{As}$ serve a dual purpose, acting both as acceptors and as magnetic impurities, whose spins align at the ferromagnetic transition [2]. Since it is widely believed that the carriers are mediating ferromagnetic interaction, ferromagnetism in DMS is called carrier-induced ferromagnetism and several theories addressing the mechanism are already available [3]. Some mean-field theories, based on the RKKY interaction [4-5] have succeeded in explaining some characteristic and transport properties of DMS's. However, further results show that for a low doping level the RKKY interaction between localized spins is insufficient [6-7]. In this case the impurity band model, where $p$ holes move around interacting with localized spins at $\mathrm{Mn}$ sites through the antiferromagnetic exchange interaction, is a widely used model for (III,Mn)V-type DMS [8-9]. In almost theoretical works above, however, the effects of super-exchange interaction between d-electrons in Mn have been neglected. 
From the application point of view, the low Curie temperature of the investigated DMS represent a serious problem, and many efforts have been devoted to find DMS with higher $T_{c}$. On the other hand, the temperature dependence $m(T)$ of the spontaneous magnetization possesses many important characteristics, such as concavity/convexity of the curve, value of saturation magnetization, etc., so its study has a very high potential for elucidating the physics behind DMS ferromagnetism in real systems. The purpose of this paper is to calculate the magnetization as a function of temperature for a wide range of model parameters including the antiferromagnetic coupling constant between magnetic impurities. We show that for a degenerate carrier system the suppression of the magnetization is sensitive to the antiferromagnetic coupling constant and the impurity concentration.

\section{THE MODEL AND FORMALISM}

We consider the following model of III-V DMS $\mathrm{A}_{1-x} \mathrm{Mn}_{x} \mathrm{~B}$ where both of the exchange interaction between carrier and impurity spins, and the direct exchange interaction between magnetic impurities are taken into account

$$
H=\sum_{i j \sigma} t_{i j} a_{i \sigma}^{+} a_{j \sigma}+\sum_{i} u_{i}-J \sum_{<i j>} \vec{S}_{i} \vec{S}_{j}
$$

where $u_{i}$ is either $u_{i}^{A}$ or $u_{i}^{M}$ depending on the ion species occupying the $i$ site:

$$
u_{i}= \begin{cases}E_{A} \sum_{\sigma} a_{i \sigma}^{+} a_{i \sigma}, & i \in A \\ E_{M} \sum_{\sigma} a_{i \sigma}^{+} a_{i \sigma}-\Delta \sum_{\sigma} a_{i \sigma}^{+} a_{i \sigma}\left(\sigma S_{i}\right), & i \in M n .\end{cases}
$$

Here $a_{i \sigma}^{+}\left(a_{i \sigma}\right)$ is the creation (annihilation) operator for a carrier with spin $\sigma$ at $i$ site; $\vec{S}_{i}$ denotes the spin of localized impurity at $i$ site ; $\Delta$ is the effective coupling constant between the localized spin and itinerant spin; $J$ is the coupling constant between the neighboring localized impurity spins, which depends on their distance and for the AF exchange interaction case $J<0$. To consider the effect of the direct exchange interaction between magnetic impurities on magnetization, we simply the problem, dividing equation (1) into the impurity term and the itinerant carrier term.

$$
\begin{gathered}
H_{i m p}=-\sum_{i} h S_{i}^{z}-J \sum_{<i j>} S_{i}^{z} S_{j}^{z}, \\
H_{c a r r}=\sum_{i j \sigma} t_{i j} a_{i \sigma}^{+} a_{j \sigma}+\sum_{i} u_{i},
\end{gathered}
$$

where $h$ is the field induced by the polarization of the carrier spins. In this study we treat the localized spin as the Ising spin $\left(S_{i}^{z}= \pm 1\right)$ and treat the $H_{i m p}$ in the molecular field approximation as $S_{i}^{z} S_{j}^{z}=<S_{i}^{z}>S_{j}^{z}+<S_{j}^{z}>S_{i}^{z}-<S_{i}^{z}><S_{j}^{z}>$. Within this mean approximation, the Hamiltonian (3) becomes

$$
H_{i m p}^{M F}=N x J \gamma m^{2} / 2-\sum_{i} S_{i}^{z}(h+J \gamma m)
$$


where $N$ is the number of lattice sites, $x$ is Mn density, $m=<S_{i}^{z}>$ refers to the average magnetization per lattice site, $\gamma$ is the number of the neighboring localized impurity spins located around a given one. With simplified Hamiltonian (5), we obtain the partition function

$$
Z_{i m p}=\left(e^{-\beta J \gamma m^{2} / 2} \sum_{S^{z}= \pm 1} e^{\beta(h+J \gamma m) S^{z}}\right)^{N x},
$$

where $\beta=1 / k_{B} T$. The free energy for the localized spin system is then given as

$$
F_{i m p}=-k_{B} T \ln Z_{i m p}=N x J \gamma m^{2} / 2-N x k_{B} T \ln \left(\sum_{S^{z}= \pm 1} e^{\beta(h+J \gamma m) S^{z}}\right)
$$

We apply CPA $[8,10]$ to the Hamiltonian $(4)$. In CPA the carriers are described as independent particles moving in an effective medium of spin-dependent coherent potentials. The coherent potential $\Sigma_{\sigma}(\sigma=\uparrow, \downarrow)$ is determined by demanding the scattering matrix for a carrier at an arbitrarily chosen site embedded in the effective medium vanished on average. By using a bare semicircular noninteracting density of states (DOS) with halfbandwidth $W: \rho_{0}(z)=\frac{2}{\pi W^{2}} \sqrt{W^{2}-z^{2}}$ we obtain the following equation for the Green function for a given magnetization $m$

$$
G_{\sigma}(\omega)=\frac{1-x}{\omega-w G_{\sigma}(\omega)-E_{A}}+\frac{x(1+m) / 2}{\omega-w G_{\sigma}(\omega)-E_{M}+\Delta \sigma}+\frac{x(1-m) / 2}{\omega-w G_{\sigma}(\omega)-E_{M}-\Delta \sigma},
$$

where $w=W^{2} / 4$ and $\sigma= \pm 1$.

The Eq. (8) is easily transformed into a quartic equation for $G_{\sigma}(\omega)$ and it is solved analytically by using Farrari method. Throughout this work, we assume that the carriers are degenerate. Then the carrier density and energy can be expressed as

$$
\begin{aligned}
n & =\int_{-\infty}^{\mu}\left(\rho_{\uparrow}(\omega)+\rho_{\downarrow}(\omega)\right) d \omega, \\
E_{\text {carr }}(m) & =\int_{-\infty}^{\mu} \omega\left(\rho_{\uparrow}(\omega)+\rho_{\downarrow}(\omega)\right) d \omega,
\end{aligned}
$$

where $\mu$ is the chemical potential and $\rho_{\sigma}(\omega)=-\frac{1}{\pi} \Im G_{\sigma}(\omega)$ is the DOS with spin $\sigma$. The free energy per site of the system (1) at temperature $T$ is given as

$$
F(m)=E_{\text {carr }}(m)+h m x+x J \gamma m^{2} / 2-x k_{B} T \ln \left(\sum_{S^{z}= \pm 1} e^{\beta(h+J \gamma m) S^{z}}\right) .
$$

By minimizing $F$ with respect to $m$ we obtain the following equation for $h$

$$
h=-\frac{1}{x} \frac{d E_{\text {carr }}(m)}{d m} .
$$

By using the Weiss molecular field theory, each impurity spin feels an effective field $h+J \gamma m$ and thus we have

$$
m=\tanh \beta(h+J \gamma m) .
$$

Equations (8)-(10), (12) and (13) form a set of self-consistent equations for $\mu$ and $m$ for a given set of parameter values $x, n, \Delta, J, E_{A}, E_{M}$ and $T$. 


\section{NUMERICAL RESULTS AND DISCUSSION}

Through this work we take $E_{A}$ as the origin $(=0)$ and $W$ as the unit of energy, $\gamma=6$ for simple cubic lattice. Before numerical solving the equations (12) -(13), let us briefly consider limiting case. In the absence of the direct exchange interaction between magnetic impurities, setting $J=0, \Delta=-0.4$ and $E_{M}=-0.3$ in (13) we reproduce the CPA result for the magnetization of $\mathrm{Ga}_{1-x} \mathrm{Mn}_{x} \mathrm{As}$ obtained by Takahashi et al [11]. In addition, since $\tanh x$ is an increasing function from Eq. (13) it is easily seen that taking into account the antiferromagnetic interaction between localized spins $(J<0)$ leads to decreasing the magnetization $m(T)$.

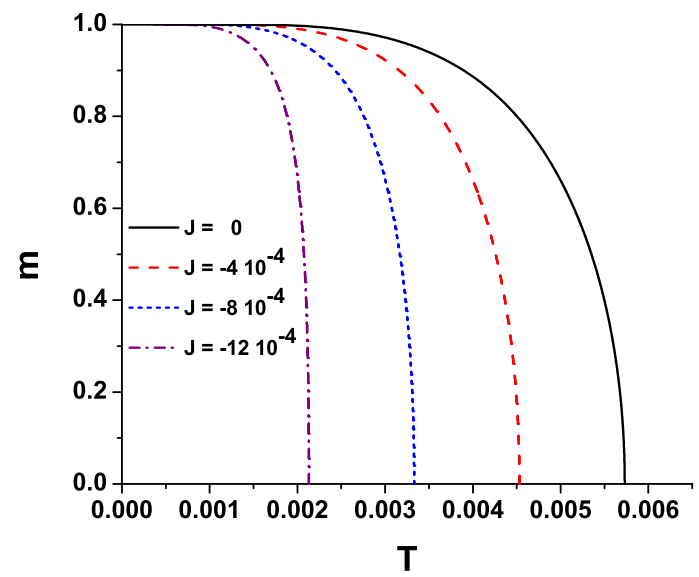

Fig. 1. Temperature dependent magnetization for various antiferromagnetic couplings for $x=0.05, n=0.025, E_{M}=-0.2, \Delta=-0.3$.

We turn now to present our numerical results. In Fig. 1, we show our calculated magnetization of the local moments as a function of temperature for different values of $J=$ $0,-4,-8$ and $-12.10^{-4}$, for $x=0.05, n=0.025, E_{M}=-0.2$ and $\Delta=-0.3$. One can see that the ferromagnetism is always preferable at low temperatures and for fixed $x, n, E_{M}, \Delta$ and $T$ the magnetization decreases with increasing $|J|$. This constant depends on the distance between two neighbour impurities, so it depends on the impurity concentration $x$. Unfortunately, as noted in [12], non of $J$ neither $x, n, E_{M}$ of our model is directly experimentally measurable. That is why a detailed comparison between our result and experiment cannot be done. Here we choose the magnitude of $J$ in the same order as in Ref.[12]. These results indicate that the neighboring magnetic impurities not only couple anti-ferromagnetically to each other but also reduce the carrier-induced ferromagnetic interaction.

In Fig. 2, the temperature dependence of magnetization is plotted for $x=0.05, n=$ $0.025, E_{M}=-0.2, J=-4.10^{-4}$ and for several values of $\Delta=-0.3,-0.4$ and -0.6 . Here, the fact that our $m(T)$ and $T_{c}$ increases with increasing $|\Delta|$ for all $\Delta$ is due to the Weiss mean field theory. Fig. 3 displays the change of the magnetization with the 


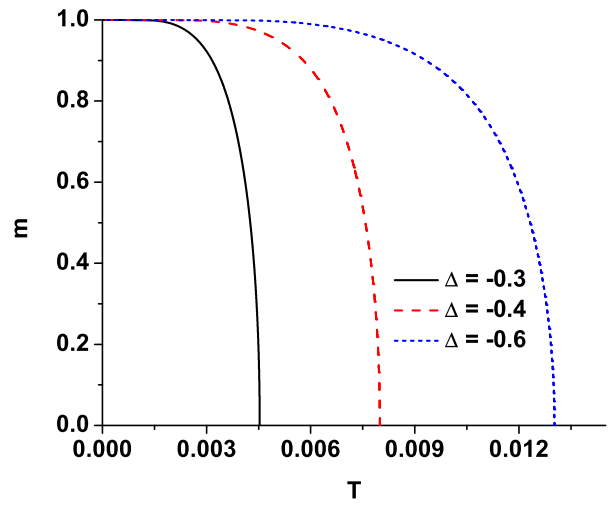

Fig. 2. Temperature dependent magnetization for various effective coupling constants for $x=0.05, n=0.025, E_{M}=-0.2, J=$ $-4 \times 10^{-4}$

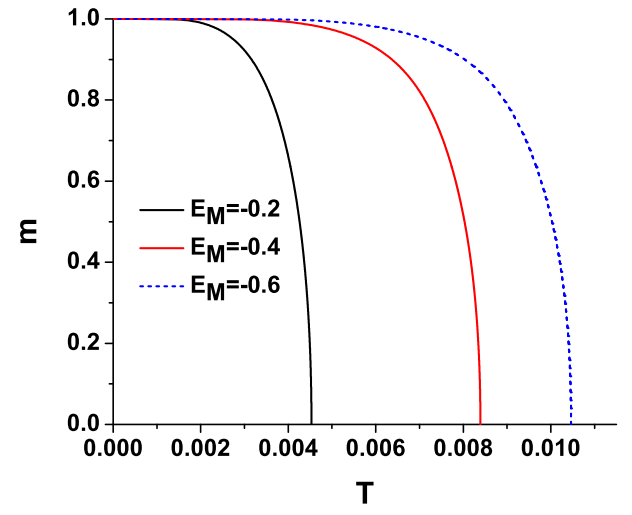

Fig. 3. Magnetization for different values of nonmagnetic potential for $x=0.05, n=$ $0.025, \Delta=-0.3, J=-4.10^{-4}$.

change of nonmagnetic potential. Comparing with the curves in Fig. 2 it is clear that $E_{M}$ simply renormalizes the effective value of $\Delta$. Fig. 4 and Fig. 5 show the magnetization $m(T)$ for various value of $x$ and $n$. We find that the magnetization is sensitive to the impurity concentration $x$ and $m(T)$ is maximized for the carrier density roughly half of the concentration of localized spins $(n / x \approx 0.5)$ which agrees with $[9,12]$. However, unlike Ref. [12] where the magnetization curve is concave for low carrier densities, our curve is convex for all values of $n$.

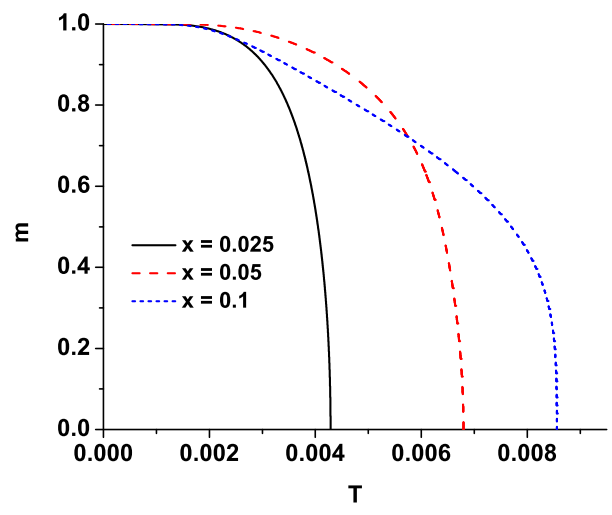

Fig. 4. Temperature dependent magnetization for various magnetic impurity concentrations for $n=0.02, E_{M}=-0.3, \Delta=$ $-0.3, J=-4.10^{-4}$.

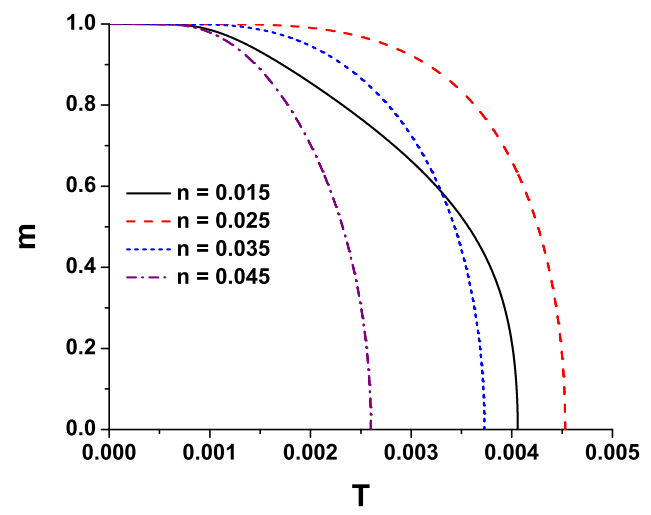

Fig. 5. Magnetization for various values of carrier density for $x=0.05, E_{M}=-0.2, \Delta=$ $-0.3, J=-4.10^{-4}$. 
To summarize, applying the CPA and mean-field approximation we have studied the effects of the direct exchange interaction between magnetic impurities on magnetization in DMS (III,Mn)V-type. The magnetization as a function of temperature for a wide range of model parameters is calculated and discussed. We have shown that for a degenerate carrier system the suppression of the magnetization is sensitive to the antiferromagnetic coupling constant and the impurity concentration. This result also implies that the superexchange interaction between d-electrons in $\mathrm{Mn}$ has a tendency to reduce $T_{c}$ which will be investigated in the near future.

\section{ACKNOWLEDGMENTS}

This work is supported by the National Foundation for Science and Technology Development (NAFOSTED).

\section{REFERENCES}

[1] G. Schmidt et al., Phys. Rev. B62 (2000) R4790.

[2] H. Ohno, J. Magn. Magn. Mater. 200 (1999) 110.

[3] T. Jungwirth et al., Rev. Mod. Phys. 78 (2006) 809.

[4] G. Zarand and B. Janko, Phys. Rev. Lett., 89 (2002) 047201.

[5] T. Dietl, H. Ohno and F. Matsukura, Phys. Rev., B63 (2001) 195205.

[6] G. A. Fiete at al., Phys. Rev., B71 (2005) 115202.

[7] R. Bouzerar et al. Phys. Rev., B73,(2006) 024411.

[8] M. Yagi and Y. Kayanuma, J. Phys. Soc. Jpn., 71 (2002) 2010.

[9] M. Takahashi and K. Kubo, J. Phys. Soc. Jpn., 72 (2003) 2866.

[10] Hoang Anh Tuan and Le Duc Anh, Commun. Phys., 15 (2005) 199.

[11] M. Takahashi, N. Furukawa and K. Kubo, J. Mag. Magn. Mag. Mater. 272-276 (2004) 2021.

[12] S. Das Sarma, E.H. Hwang, and S. A. Kaminski, Phys. Rev. B67 (2003) 155201.

Received 05 September 2011. 